УДК 622.276.53.054.23:621.67-83

\title{
ЭКСПЕРИМЕНТАЛЬНЫЕ ИССЛЕДОВАНИЯ РАБОТЫ ПНЕВМОПРУЖИННОГО КОМПЕНСАТОРА ДАВЛЕНИЯ С КВАЗИНУЛЕВОЙ ЖЕСТКОСТЬЮ
}

\author{
Зотов Алексей Николаевич', \\ a-zot2@yandex.ru
}

Думлер Елена Борисовна',

dumler08@mail.ru

Уразаков Камил Рахматуллович', UrazakK@mail.ru

\section{Вахитова Роза Ильгизовна²,}

roza-w@mail.ru

\section{Думлер Олег Юрьевич mail@ntehn.ru}

\author{
Тугунов Павел Михайлович', \\ info@rusoil.net \\ 1 Уфимский государственный нефтяной технический университет, \\ Россия, 450062, г. Уфа, ул. Космонавтов, 1. \\ 2 Альметьевский государственный нефтяной институт, \\ Россия, 423450, г. Альметьевск, ул. Ленина, 2. \\ 3000 «Новые технологии», \\ Россия, 422980, г. Чистополь, ул. Карла Маркса, 168 с.
}

Актуальность исследования обусловлена воздействием вибрации на элементы установки погружного электроцентробежного насоса. Наличие вибрационных процессов увеличивает количество преждевременных отказов узлов установки. Одной из причин проявления вибрационного воздействия на электроцентробежную насосную установку являются перепады давления на выкиде погружного электроцентробежного насоса, при этом частоты таких перепадов невелики.

Цель: разработка лабораторной установки, которая имитирует колебательные процессы, происходящие на выкидной линии электроцентробежного погружного насоса в нефтяной добывающей скважине.

объекты: модель пневмопружинного компенсатора давления с квазинулевой жесткостью, конструктивно представляющего собой пневматическую пружину, внутри которой расположен пакет из пяти последовательно установленных тарельчатых пружин. Методы: численные решения дифференциальных уравнений, теория колебаний, математический анализ, математическая статистика, методы математического моделирования.

Результаты. Разработана лабораторная установка, позволяющая провести исследования модели пневмопружинного компенсатора давления с квазинулевой жесткостью, конструктивно представляющего собой пневматическую пружину, внутри которой расположен пакет из пяти последовательно установленных тарельчатых пружин. Система с квазинулевой жесткостью представляет собой совокупность пневмопружины, имеющей силовую характеристику с рабочим участком положительной жесткости, и пакета последовательно соединенных тарельчатых пружин, имеющего силовую характеристику с рабочим участком отрицательной жесткости. Экспериментально подобраны параметры тарельчатых пружин таким образом, чтобы получить силовую характеристику с участком квазинулевой жесткости при заданном давлении. Результатом исследований на лабораторном стенде стало совпадение с заданной погрешностью замеров размаха колебаний и теоретически предсказанных результатов, что позволило доказать правильность теоретических выводов.

\section{Ключевые слова:}

Установка электроцентробежного погружного насоса, лабораторная установка, пневмопружинный компенсатор давления пневмопружина, квазинулевая жесткость, отрицательная жесткость, тарельчатые пружины.

\section{Введение}

Установки электропогружных центробежных насосов (УЭЦН) на современном этапе являются основным оборудованием для добычи нефти на нефтяных промыслах Российской Федерации.

Одной из существенных причин колебательного воздействия на насосную погружную установку являются скачки давления на выкидной линии
УЭЦН, при этом частоты этих скачков невелики $[1,2]$. Большая часть используемых насосных компенсаторов - это пневматические компенсаторы, представляющие собой газовые колпаки [3]. Для получения эффекта от пневмокомпенсатора такого типа в электроцентробежных насосах (ЭЦН) в скважинных условиях длина пневмоколпака будет составлять десятки метров, что технически достаточ- 
но сложно выполнить. Эффективным методом гашения низкочастотных колебаний является использование системы с квазинулевой жесткостью [4-14], которая в 70-х гг. ХХ столетия была предложена профессором П.М. Алабужевым для виброзащиты [15]. На основе пневмопружины и пакета последовательно соединенных тарельчатых пружин, расположенного внутри пневмопружины и подпирающего ее поршень, разработан пневмопружинный компенсатор давления с рабочим участком, имеющим силовую характеристику с квазинулевой жесткостью большой длины $[1,2,16]$.

\section{Методика испытаний системы}

\section{с квазинулевой жесткостью}

Предлагаемая лабораторная установка состоит из пневмопружины, представляющей собой стальной цилиндр с поршнем, в которой расположен пакет из последовательно соединенных тарельчатых пружин (рис. 1). Параметры тарельчатых пружин рассчитали так, чтобы получилась силовая характеристика с участком квазинулевой жесткости при условии, что задано давление в пневмопружине.

В цилиндре - 7 расположен пакет из пяти последовательно соединенных тарельчатых пружин - 15, который жестко связан с поршнем - 6 с помощью втулки - 22. Для исключения смещения тарельчатых пружин при их осевом перемещении между ними устанавливают шайбы - 14. Для воз- буждения вертикальных колебаний к набору грузов - 8 присоединен вибратор, который состоит из двигателя постоянного тока - 9, связанного с эксцентриком - 10. Грузы через специальный шток 2 жестко соединены с поршнем - 6 .

Участок квазинулевой жесткости получен после нагружения предлагаемого лабораторного стенда расчетным заданным весом на силовой характеристике этой системы. Внутренний диаметр цилиндра, где располагается пакет последовательно соединенных тарельчатых пружин, равен 0,0380 м; длина хода поршня $\mathrm{H}=0,1230$ м (рис. 1). Внешний диаметр пакета тарельчатых пружин для цилиндра принимаем $D=0,0370$ м для того, чтобы пружины при сжатии не заклинивали, а внутренний диаметр $d=0,0230$ м. Пакет тарельчатых пружин включает в себя пять пружин - 1 и стальные шайбы - 2 (рис. 2, б) [17].

Решено изготовить тарельчатые пружины (рис. 2, б) из пластического материала типа Flex, имеющего значение модуля упругости $E=74 \cdot 10^{6} \mathrm{H} / \mathrm{m}^{2}$, на $3 \mathrm{D}$ принтере марки $\operatorname{Rec}$ [17]. В виду того, что тарельчатые пружины изготовлены из пластика, толщину конуса пружин принимаем $s=0,0020$ м (рис. $2, a)$. В случае использования более тонкой толщины конуса пружины при сжатии происходит ее изгиб. Высоту конуса пружины принимаем $f=0,0050$ м, для обеспечения заметной длины хода поршня $(0,0250$ м) (рис. $2, a)$. a)

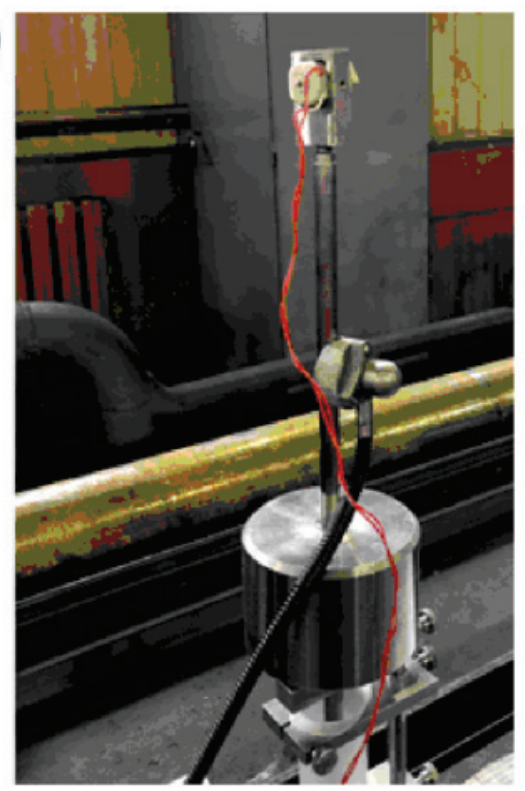

б)

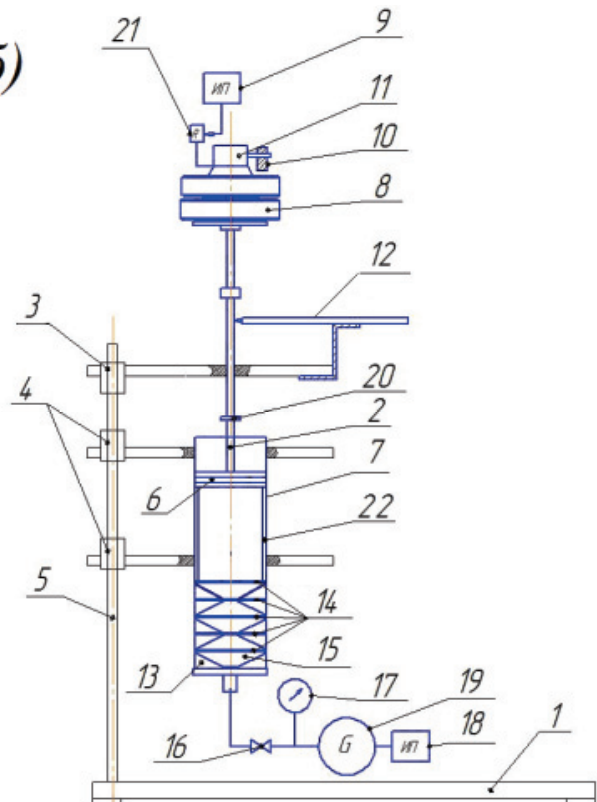

Рис.1. Лабораторная установка для проведения исследований колебаний: а) стенд для исследования низкочастотных колебаний; б) схела принципиальная: 1 - рала; 2 - шток; 3, 4 - специальные крепления; 5 - штатив; 6 - поршень; 7 - иилиндр; 8 - набор грузов; 9 - источник питания; 10 - эксиентрик; 11 - электродвигатель; 12 - неподвижный маркер; 13 - пневматическая камера; 14 - шайбы; 15 - тарельчатые пружины; 16 - кран; 17 - манометр; 18 - источник питания колпрессора; 19 - компрессор; 20 - упор; 21 - резистор переменного тока; 22 - втулка

Fig. 1. Laboratory installation for research of oscillations: a) stand for studying low-frequency oscillations; 0 ) schematic diagram: 1 is the frame; 2 is the rod; 3, 4 are the special fasteners; 5 is the tripod; 6 is the piston; 7 is the cylinder; 8 is the set of weights; 9 is the power supply; 10 is the eccentric; 11 is the electric motor; 12 is the fixed marker; 13 is the pneumatic chamber; 14 are the washers; 15 is the dish-shaped springs; 16 is the crane; 17 is the manometer; 18 is the compressor power supply; 19 is the compressor; 20 is the stop; 21 is the alternating current resistor; 22 is the bushing 

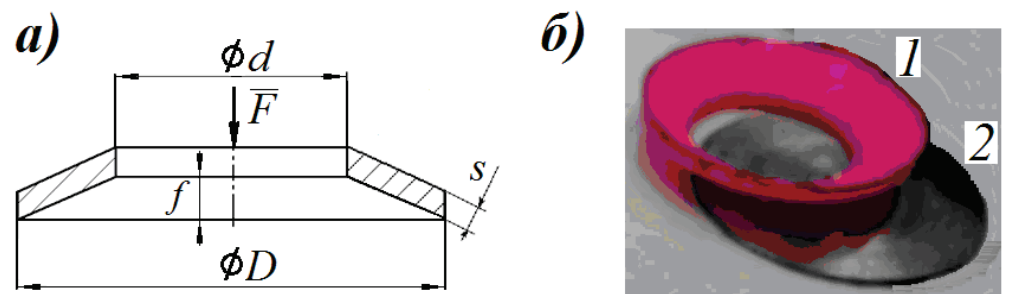

Pис. 2. Тарельчатая пружина: а) схема тарельчатой пружины: $s$ - толщина конуса тарельчатой пружины; D-внешний диалетр тарельчатой пружины; $d$-внутренний диаметр тарельчатой пружины; $f$-высота конуса тарельчатой пружины; б) фотография тарельчатой пружины: 1 - пружина тарельчатая; 2 - шайба

Fig. 2. Dish-shaped spring: a) the diaphragm of spring arrangement: $s$ is the thickness of the dish-shaped spring cone; $D$ is the outside diameter of the dish-shaped spring; $d$ is the inside diameter of the dish-shaped spring; $f$ is the height of the dish-shaped spring cone; $\sigma$ ) photograph of the dish-shaped spring: 1 is the dish-shaped spring; 2 is the washers

Формула (1) определяет восстанавливающую силу предлагаемой системы, представляющей собой пневматическую пружину, внутри которой расположен пакет из пяти последовательно установленных тарельчатых пружин.

Показатель политропы принимаем равным единице, что является справедливым для не очень быстрых движений [18]. Первое слагаемое формулы (1) выражает восстанавливающую силу пневмопружины, а второе слагаемое этой формулы восстанавливающую силу пакета из пяти последовательно установленных тарельчатых пружин.

$$
\begin{gathered}
F_{\Sigma}=\frac{p_{0} S H}{(H-x)}+8 \pi E s\left(\frac{x}{N}\right) \times \\
\left\{\begin{array}{l}
\left(f-\left(\frac{x}{N}\right)\right)\left(f-\left(\frac{x}{N}\right) / 2\right) \times \\
\times\left[\frac{(D+d)}{2(D-d)}-\frac{1}{\ln (D / d)}\right]+ \\
+s^{2} \ln \left(\frac{D}{d}\right) / 12
\end{array}\right\} \\
\times \frac{(D-d)^{2}}{(D)}
\end{gathered}
$$

где $p_{0}$ - начальное давление в пневмопружинном компенсаторе давления; $S=\frac{\pi \cdot 0,038^{2}}{4}, \mathrm{M}^{2}-$ площадь поршня; $H=0,1230$ м - высота цилиндра; $N$ количество тарельчатых пружин; $E$ - модуль упругости первого рода материала тарельчатой пружины; $s$ - толщина конуса тарельчатой пружины (рис. $2, a) ; x$ - осадка тарельчатой пружины; $f=0,0050 \mathrm{~m}$ - высота конуса тарельчатой пружины (рис. $2, a) ; D=0,0380$ м - внешний диаметр тарельчатой пружины (рис. $2, a) ; d-$ внутренний диаметр тарельчатой пружины (рис. $2, a$ ).

Подбираем параметры предлагаемой лабораторной установки $p_{0}, d, N$, входящие в формулу (1), методом последовательных приближений в режиме работы с компьютером для обеспечения приемлемой величины квазипостоянной силы не более $200 \mathrm{H}$.

Силовые характеристики, полученные по формуле (1), представлены на рис. 3 . Зависимости 1 представляют собой полученные по формуле (1) суммарные силовые характеристики. Кривые 2 представляют зависимости первого слагаемого из формулы (1) от координаты - положительная же-
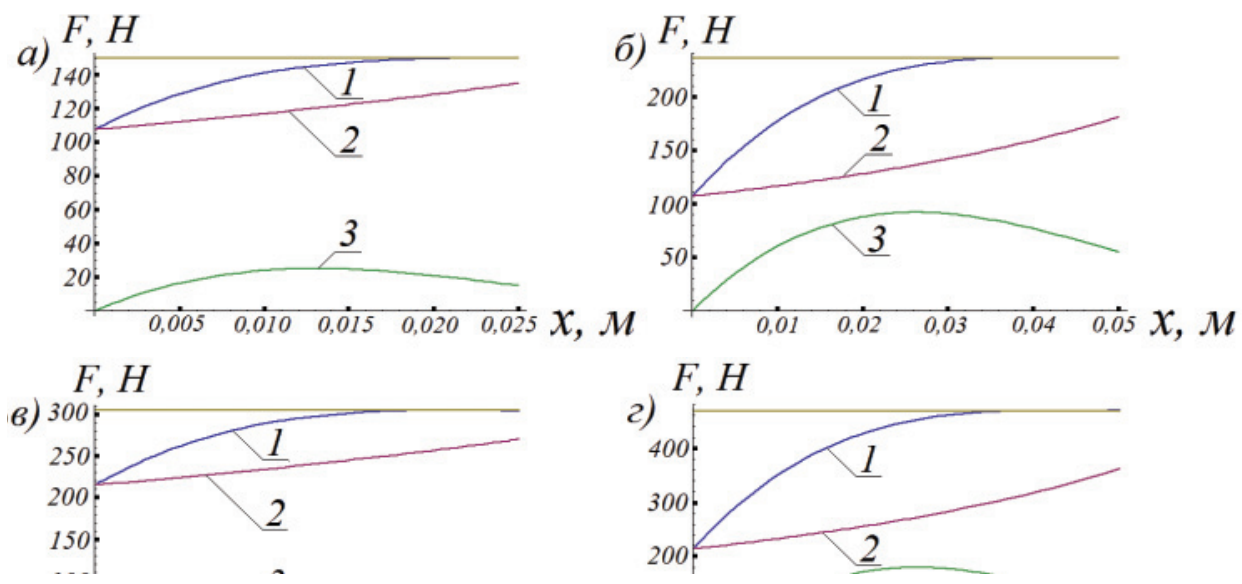

Рис. 3. Теоретические силовые характеристики лабораторной установки, полученные по формуле (1)

Fig. 3. Theoretical power characteristics of the laboratory installation, obtained by the formula (1)

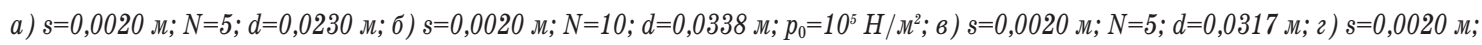
$N=10 ; d=0,0354 m ; p_{0}=2 \cdot 10^{5} \mathrm{H} / \mathrm{M}^{2}$ 


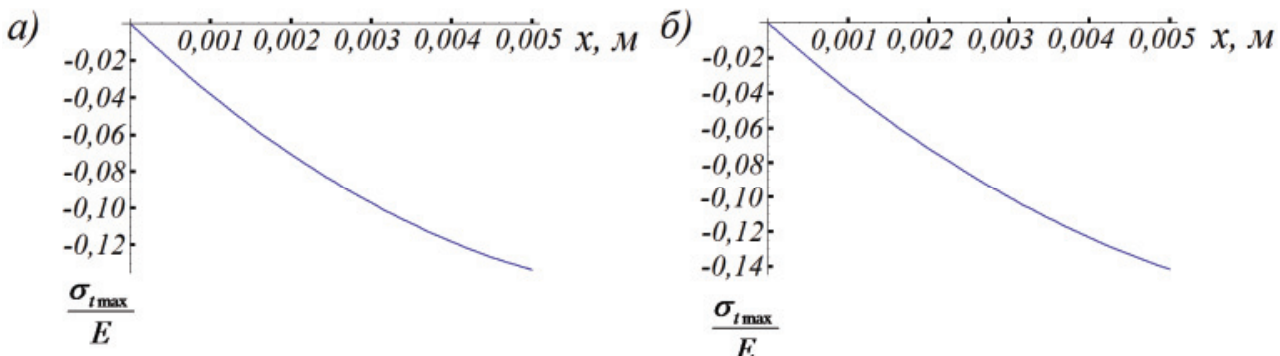

Pис. 4. Зависимости критерия подобия $\sigma_{t} /$ Е от осадки пружины: а) отношение $\sigma_{t} /$ Е для тарельчатых пружин, рассчитанных для лабораторной установки: $D=0,037 ., d=0,023 \mathrm{~m} ; f=0,005 \mathrm{~m} ; s=0,002 \mathrm{~m} ;$ б) отношение $\sigma_{t} /$ E для тарельчатых пружин, рассчитанных для реальных скважинных условий: $D=0,056 \mathrm{~m} ; d=0,048 \mathrm{~m} ; \mathrm{f}=0,005 \mathrm{~m} ; \mathrm{s}=0,003 \mathrm{~m}$

Fig. 4. Dependence of the similarity criterion $\sigma_{t} / E$ on the spring draft: a) attitudes $\sigma_{t} / E$ for dish-shaped springs designed for laboratory use: $D=0,037 \mathrm{~m} ; d=0,023 \mathrm{~m} ; f=0,005 \mathrm{~m} ; \mathrm{s}=0,002 \mathrm{~m} ; \sigma)$ attitudes $\sigma_{t} / \mathrm{E}$ for dish-shaped springs designed for real borehole conditions: $D=0,056 \mathrm{~m}$; $d=0,048 \mathrm{~m} ; \mathrm{f}=0,005 \mathrm{~m} ; \mathrm{s}=0,003 \mathrm{~m}$

сткость. Кривые 3 представляют зависимости второго слагаемого от координаты - отрицательная жесткость. Из рис. 3, в, г видно, что участки квазинулевой жесткости (величины квазипостоянных сил) более $200 \mathrm{H}$, таким образом, масса грузов не должна превышать 20 кг для удобства проведения эксперимента. В результате анализа данных был принят вариант $a$.

При изготовлении лабораторной установки критерием подобия принято соотношение $\sigma / E$, применяемое в том случае, когда материалы промышленного образца и установки различны. Были подобраны такие параметры лабораторной установки, которые позволили обеспечить приемлемую величину квазипостоянной силы [19].

По формуле (2) определяется отношение [20]:

$$
\frac{\sigma_{t \max }}{E}=-\frac{4 x}{(D-d)^{2}}\left[\left(\frac{2 c}{d}-1\right)\left(f-\frac{x}{2}\right)+\frac{s}{2} \frac{D-d}{d}\right] \text {, }
$$

где $c=\frac{D-d}{2 \ln \frac{D}{d}} ; \sigma_{t}-$ максимальное напряжение в меридиональном сечении диска тарельчатой пружины; $E$ - модуль упругости материала пружины.

Как следует из правой части формулы (2), выбор материала тарельчатой пружины не важен, важны параметры пружины (модуль упругости не входит в нее).

На рис. 4 представлены зависимости критерия подобия $\sigma_{t} / E$ от осадки пружины х для тарельчатых пружин, рассчитанных для лабораторной установки (график $a$ ), а для тарельчатых пружин, рассчитанных для реальных скважинных условий - график б. Размеры тарельчатой пружины для графика б были рассчитаны ранее в работе [2]. Из рис. 4 видно, что величины критерия подобия являются соизмеримыми в обоих вариантах, это говорит о правильном выборе размеров лабораторной установки.

Из-за силы сухого трения сложно демонстрировать квазинулевую жесткость. Определили силу сухого трения между цилиндром и поршнем. Для этого замерили время спуска поршня с высоты
$H=x_{m}=0,123$ м при воздействии веса $m \cdot g$, где $m=0,656$ кг - масса поршня со штоком; $g=9,81 \mathrm{~m} / \mathrm{c}^{2}-$ ускорение свободного падения.

Формула, по которой рассчитывалась сила трения, следующая (элементарные выкладки опущены):

$$
F_{\text {тр }}=m \cdot g-2 m \cdot x_{m} / t_{*}^{2},
$$

где $t_{*}$ - время спуска поршня.

Среднее время спуска поршня по восьми проведенным опытам оказалось равным $t_{*}=0,25 \mathrm{c}$, тогда $F_{\text {тр }} \approx 3,85 \mathrm{H}$. Сила трения, рассчитанная по формуле (3), принята равной $4 \mathrm{H}$, так как из-за наличия избыточного давления в полости цилиндра сила трения может быть несколько выше.

На рис. 5 представлена силовая характеристика пакета пяти тарельчатых пружин, полученная теоретическим путем, с участком, имеющим отрицательную жесткость.

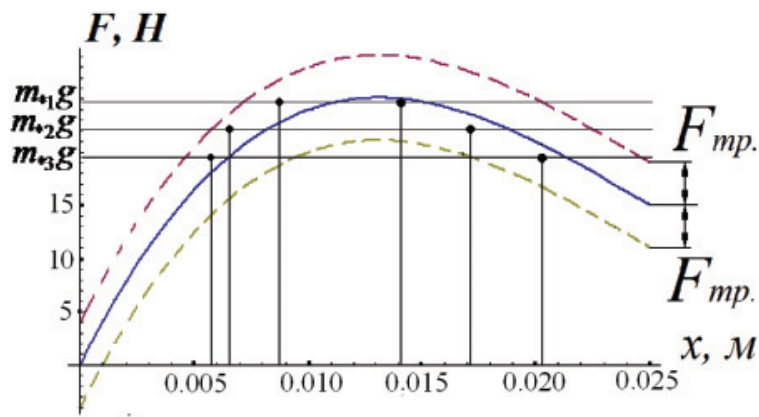

Pис. 5. Силовая характеристика тарельчатых пружин, полученная с учетом $F_{\text {тр }}=4 \mathrm{H}$

Fig. 5. Power characteristic of the dish-shaped springs, obtained with regard to $F_{\mathrm{fr}}=4 \mathrm{~N}$

Теоретическая зависимость, определяемая вторым слагаемым формулы (1), показана сплошной линией синего цвета (рис. 5), а пунктирные линии показывают зависимость между восстанавливающей силой пакета пяти тарельчатых пружин и перемещением с учетом силы сухого трения $F_{\text {тр }}=4 \mathrm{H}$.

При возвратно-поступательном перемещении поршня сила трения $F_{\text {тр }}$ то добавляется к теоретической характеристике (второе слагаемое уравне- 
ния (1) $+F_{\text {тр }}$ ), то вычитается из неё (второе слагаемое уравнения (1) $\left.-F_{\text {тр }}\right)$, и в результате образуется петля гистерезиса, которая показана верхней и нижней пунктирными линиями (рис. 5). Силовая теоретическая характеристика с участком отрицательной жесткости представленна в диапазоне примерно от $x \approx 0,013$ м до $x=0,025$ м. Это значит, что определенному значению восстанавливающей силы $F$ будут соответствовать две координаты $x$. Пакет пяти тарельчатых пружин нагрузили разными весами: $m_{* 1} g, m_{* 2} g, m_{* 3} g$ (рис. 5). Вес грузов выбирался с условием попадания в область полученной силовой характеристики для возможности тарирования лабораторной установки.

Взяли массы: $m_{*_{1}}=2,000 \mathrm{\kappa}, m_{*_{2}}=2,250 \mathrm{\kappa г}$, $m_{* 3}=2,500$ кг. Это массы грузов -8 (рис. 1) в сумме с массой поршня и штока. Эксперименты проводились по следующему алгоритму. Замеряли перемещения поршня $x_{1}$ при нагружении его весами $m_{* 1} g$; $m_{* 2} g ; m_{* 3} g$, далее проводили полное сжатие пакета пяти тарельчатых пружин и после освобождения пакета пяти тарельчатых пружин замеряли второе перемещение поршня $x_{2}$ для одного и того же веса. Массы грузов с учетом вычета массы штока следующие:

$$
\begin{aligned}
& m_{* 1}-m_{\text {шा }}=2,000-0,656=1,344 \kappa \Gamma ; \\
& m_{* 2}-m_{\text {шा }}=2,250-0,656=1,594 \kappa \Gamma ; \\
& m_{* 3}-m_{\text {шा }}=2,500-0,656=1,844 \kappa \Gamma .
\end{aligned}
$$

Провели восемь опытов, с точностью замеров до 0,5 мm.

Результаты экспериментов для массы $m_{* 1}=2,000$ кг приведены в табл. 1 .

таблица 1. Перемещения поршня

Table 1. Piston movements

\begin{tabular}{|c|c|c|c|c|c|c|c|c|}
\hline $\begin{array}{c}\text { Номер варианта } \\
\text { Version no. }\end{array}$ & 1 & 2 & 3 & 4 & 5 & 6 & 7 & 8 \\
\hline$x_{1}$, мм (mm) & 8,5 & 9 & 8 & 9 & 8,5 & 8,5 & 9 & 9 \\
\hline$x_{2}$, мм (mm) & 16 & 17 & 17 & 16,5 & 16,5 & 17 & 17 & 17 \\
\hline
\end{tabular}

Результаты экспериментов для массы $m_{* 2}=2,250$ кг приведены в табл. 2.

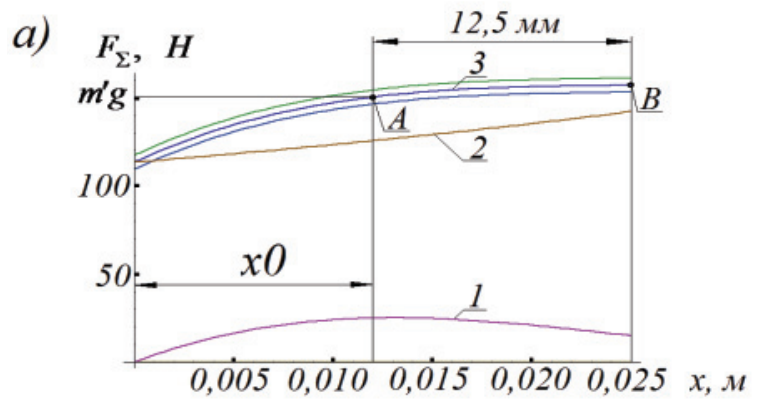

Таблииа 2. Перемещения поршня

Table 2. Piston movements

\begin{tabular}{|c|c|c|c|c|c|c|c|c|}
\hline $\begin{array}{c}\text { Hoмер варианта } \\
\text { Version no. }\end{array}$ & 1 & 2 & 3 & 4 & 5 & 6 & 7 & 8 \\
\hline$x_{1}$, мм (mm) & 6,5 & 6,5 & 7 & 6 & 6,5 & 7 & 6 & 6,5 \\
\hline$x_{2}$, мм (mm) & 20 & 20,5 & 20,5 & 20 & 20 & 20,5 & 20,5 & 19 \\
\hline
\end{tabular}

Результаты для массы $m_{* 3}=2,500$ кг приведены в табл. 3.

Таблица 3. Перемещения поршня

Table 3. Piston movements

\begin{tabular}{|c|c|c|c|c|c|c|c|c|}
\hline $\begin{array}{c}\text { Hoмер варианта } \\
\text { Version по. }\end{array}$ & 1 & 2 & 3 & 4 & 5 & 6 & 7 & 8 \\
\hline$x_{1}$, мм (mm) & 5,5 & 6 & 7 & 6 & 5,5 & 6 & 6 & 5 \\
\hline$x_{2}$, мм (mm) & 23 & 24 & 23,5 & 24 & 23 & 23 & 24 & 23,5 \\
\hline
\end{tabular}

Средние значения перемещений (табл. 1-3) отмечены точками и, как видно из графика рис. 5, попадают в петлю гистерезиса, это говорит о сходимости результатов эксперимента и опыта.

На рис. 6, а показана силовая характеристика, полученная теоретическим путем, с учетом силы сухого трения. Чтобы попасть на участок с квазинулевой жесткостью необходимо систему нагружать некоторым весом $m^{\prime} g$, в этом случае начало координат силовой характеристики сместится приблизительно на середину с координатой $x 0$.

Величину массы $m^{\prime}$ определили визуально (рис. 6, a), она равна, учитывая массу штока, $m^{\prime} \approx 15,200$ кг. Нагрузив лабораторный стенд весом $m^{\prime} g$ получили силовую характеристику $F_{\Sigma}(x)$, представленную на рис. 6 , б.Функция $F_{\Sigma}(x)$ получена аналитическим путем из формулы (1):

$$
\widehat{F}_{\Sigma}(x)=F_{\Sigma}[x-x 0]-m^{\prime} g,
$$

где $F_{\Sigma}$ - функция, определяемая по формуле (1).

Из-за присутствия сил сухого трения теоретическая силовая характеристика принимает вид петли гистерезиса. При полученной силовой характеристике свободные колебания поршня описываем, используя формулу (4), дифференциальным уравнением:

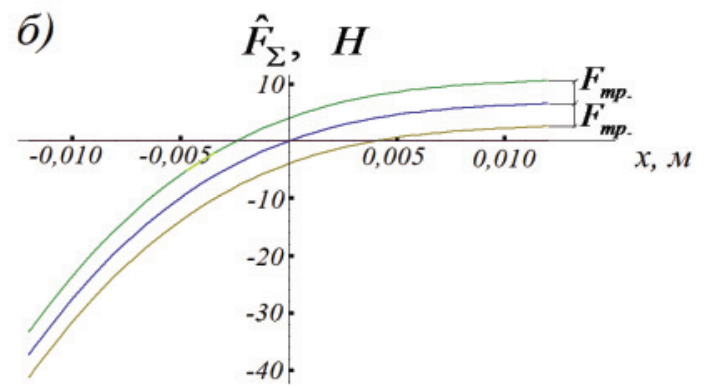

Pис. 6. Силовые характеристики лабораторной установки: а) без нагружения: 1 - силовая характеристика пакета тарельчатых пружин; 2 - силовая характеристика пневмопружины; 3 - сулмарнал силовая характеристика; $x 0$ - координата точки A (начало участка, илеющего квазинулевую жесткость); б) после нагружения весом m'g ( $F_{\text {тр }}$ с сила сухого трения)

Fig. 6. Power characteristics of laboratory installation: a) without loading: 1 is the power characteristic of the dish-shaped springs package; 2 is the power characteristic of a pneumospring; 3 is the total power characteristic; $x 0$ is the coordinate of point $A$ (the beginning of the section having quasi-zero rigidity ); $b$ ) after loading by weight ( $F_{\mathrm{fr}}$ is the force of dry friction) 


$$
m^{\prime} \cdot \ddot{x}=-\left(F_{\Sigma}[x-x 0]-m^{\prime} g\right)-F_{\mathrm{rp}} \cdot \operatorname{sign}[\dot{x}] .
$$

Начальные условия: при $\dot{x}_{0}=0, x_{0}=0,0100 \mathrm{~m}$.

Решение дифференциального уравнения (5) при различном значении сил сухого трения $F_{\text {тр }}$ представлено на рис. 7.
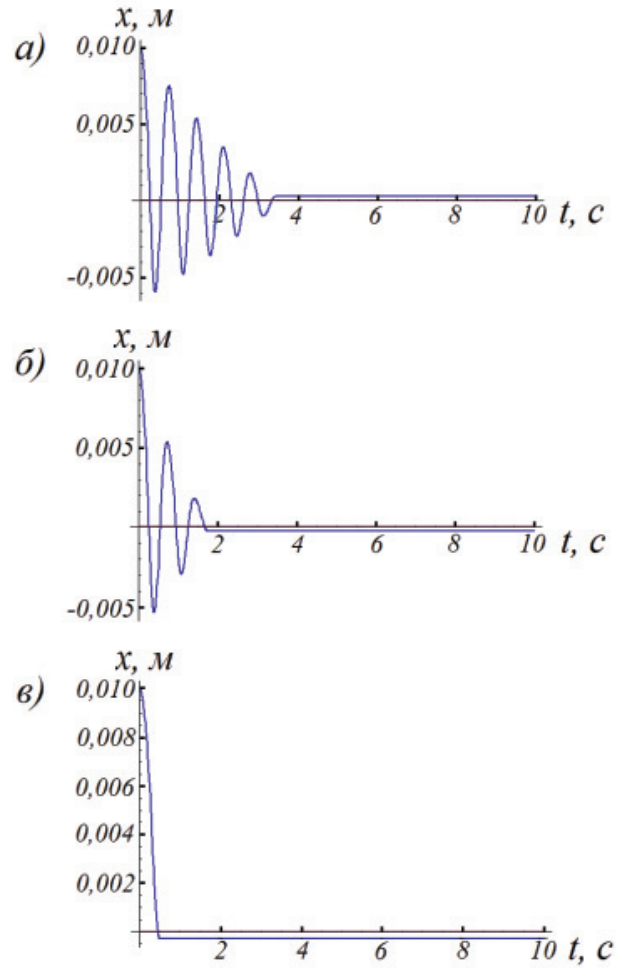

Рис. 7. Решение дифференциального уравнения (5): а) $F_{\mathrm{rp}}=0,5 \mathrm{H}$; б) $F_{\mathrm{Tp}}=1 \mathrm{H}$; в) $F_{\mathrm{Tp}}=4 \mathrm{H}$

Fig. 7. Solution of the differential equation (5): a) $F_{\mathrm{fr}}=0,5 \mathrm{~N}$; $\sigma$ ) $F_{\mathrm{fr}}=1 N$; 6$) F_{\mathrm{fr}}=4 \mathrm{~N}$

Из рис. 7 видно, что при $F_{\text {тр }}=4$ Н пропадают затухающие колебания, то есть весьма затруднительно подтвердить экспериментальным путем су- ществование полученной силовой характеристики, замеряя перемещение поршня после его отклонения на определенную величину, например, на $1 \mathrm{~cm}$.

Изменяя массу, длину и частоту вращения эксцентрика - 10 (рис. 1), была задана необходимая возмущающая сила, которой будет соответствовать расчетная амплитуда вынужденных колебаний.

В случае совпадения расчетных и экспериментальных значений амплитуд колебаний можно будет считать доказанным наличие расчетной силовой характеристики с участком квазинулевой жесткости у лабораторного стенда.

Дифференциальное уравнение (6) описывает вынужденные колебания поршня и штока и имеет вид:

$m^{\prime} \ddot{x}=F_{0} \cos [p t]-\left(F_{\Sigma}[x-x 0]-m^{\prime} g\right)-F_{\mathrm{rp}} \operatorname{sign}[\dot{x}],(6)$

где $p$ - частота вынужденных колебаний; $F_{0}$ - амплитуда возмущающей силы; $F_{\text {тр }}=4 \mathrm{H}$ - сила сухого трения.

Дифференциальное уравнение (6) решали численным методом. Решения дифференциального уравнения (6) при $m^{\prime} \approx 15,200$ кг; $F_{\text {тр }}=4 \mathrm{H}$ и частоте вращения $p=30 \mathrm{c}^{-1}(a, \sigma, z, \partial, e)$ и $p=50 \mathrm{c}^{-1}($ ( ) представлены на рис. 8. Из-за наличия сил сухого трения вынужденные колебания оказались невозможны при любом значении $F_{0}$. Как следует из рис. 8 , колебания появляются только при $F_{0}=8 \mathrm{H}$ на частотах $p=30 \mathrm{c}^{-1}$ и $p=50 \mathrm{c}^{-1}$.

Возмущающая сила в предлагаемом лабораторном стенде получена благодаря эксцентрику длиной $e$, который вращается с постоянной угловой скоростью $p$, изменяющейся в широком диапазоне с помощью двигателя постоянного тока. Силы инерции $\Phi_{1}$ и $\Phi_{2}$ определяют амплитуду возмущающей силы $F_{0}$. Силу инерции $\Phi_{1}$ прикладывают к центру эксцентрика - 1 ; силу инерции $\Phi_{2}-$ в центр стержня -2 (рис. 9). a)
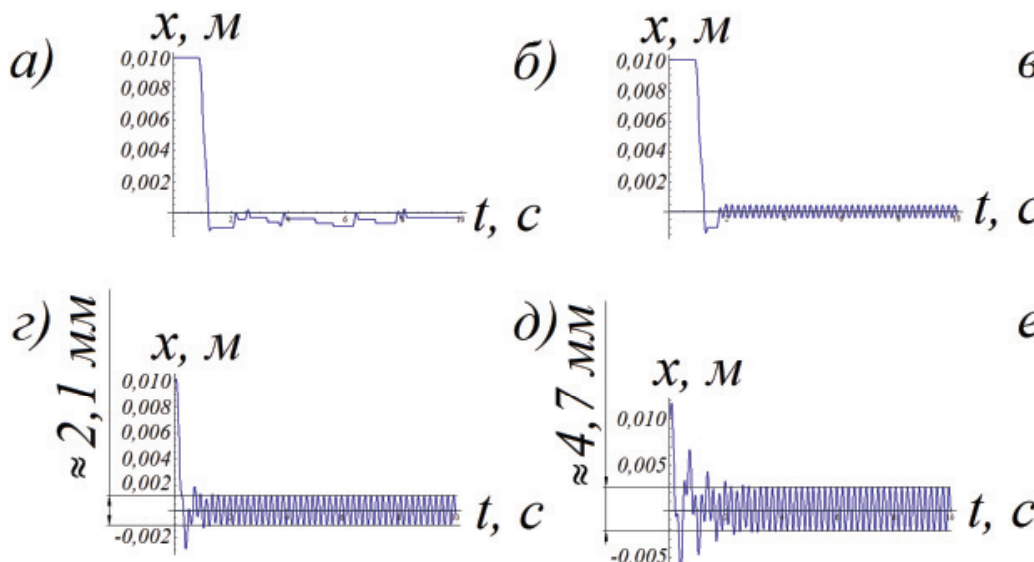

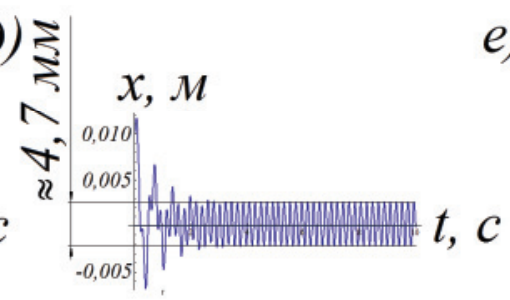

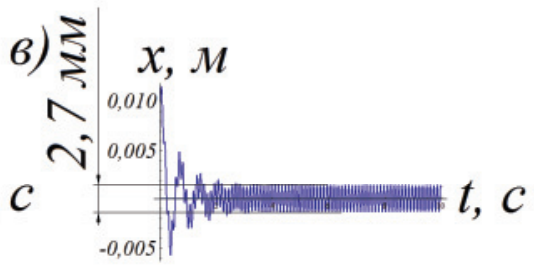

e)

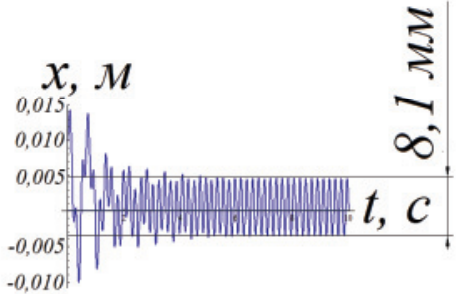

Рис. 8. Решения дифференциального уравнения (6)

Fig. 8. Solutions of the differential equation (6) 


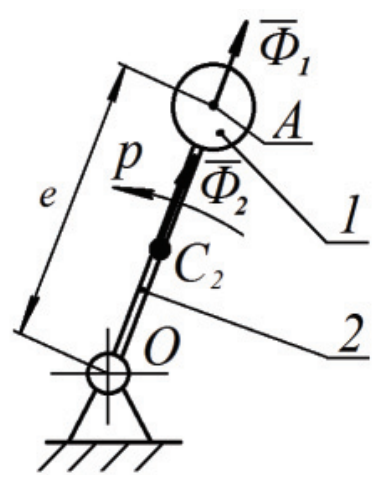

Рис. 9. Эксиентрик: 1 - эксиентрик; 2 - стержень; $m_{1}-$ масса эксиентрика; $m_{2}$ - масса стержня; $е$ - эксиентриситет (длина стержня); $р$ - постоянная угловая скорость вра щения эксиентрика

Fig. 9. Eccentric: 1 is the eccentric; 2 is the rod; $m_{1}$ is the eccentric weight; $m_{2}$ is the rod weight; $e$ is the eccentricity (rod length ); $p$ is the constant angular velocity of eccentric rota tion

Сумму сил инерции $\Phi_{1}$ и $\Phi_{2}$, равную амплитуде возмущающей силы $F_{0}$, записывают как:

$F_{0}=\Phi_{1}+\Phi_{2}=m_{1} p^{2} e+m_{2} p^{2} e / 2=p^{2} e\left(m_{1}+m_{2} / 2\right)$, где $\Phi_{1}=m_{1} p^{2} e-$ сила инерции, действующая на эксцентрик; $\Phi_{2}=m_{2} p^{2} e / 2$ - сила инерции, действующая на стержень. Дифференциальное уравнение (6) в таком случае имеет вид:

$$
\begin{gathered}
m^{\prime} \ddot{x}=p^{2} e\left(m_{1}+m_{2} / 2\right) \cos [p t]- \\
-\left(F_{\Sigma}[x-x 0]-m^{\prime} g\right)-F_{\text {тр }} \operatorname{sign}[\dot{x}],
\end{gathered}
$$

Теоретическая зависимость координаты перемещения поршня от времени, представленная на рис. 10, получена решением дифференциального уравнения (7).

Из рисунка видно, что при одинаковых значениях эксцентриситета ( $e=10$ мм) и суммы $\left(m_{1}+m_{2} / 2\right)=0,100$ кг амплитуда колебаний почти не зависит от частоты.
Размах колебаний замерялся экспериментально. Замеры осуществлялись визуально с использованием измерительного устройства - 21 (маркер), представленного на рис. 1. Погрешность этих измерений составила 0,5 мм.

Чтобы получить расчетные амплитуды возмущающих сил $F_{0}=150 \mathrm{H}, F_{0}=30 \mathrm{H}$ и $F_{0}=50 \mathrm{H}$ при $p=30 \mathrm{c}^{-1}$ (рис. 8, $2-e$ ), выбрали следующие величины эксцентриситета $e$ и масс $\left(m_{1}+m_{2} / 2\right)$ : для $F_{0}=15 \mathrm{H}$ : $\left(m_{1}+m_{2} / 2\right)=0,100$ кг; величина эксцентриситета 0,1670 м; для $F_{0}=30 \mathrm{H:}\left(m_{1}+m_{2} / 2\right)=0,200$ кг; величина эксцентриситета $-0,1660 \mathrm{~m}$; для $F_{0}=50 \mathrm{H}$ : $\left(m_{1}+m_{2} / 2\right)=0,300$ кг; величина эксцентриситета $0,1850 \mathrm{M}$.

Результаты замера амплитуды колебаний трех независимых значений амплитуды возмущающей силы $\left(F_{0}=15 \mathrm{H} ; F_{0}=30 \mathrm{H} ; F_{0}=50 \mathrm{H}\right)$ на частоте $p=30 \mathrm{c}^{-1}$ совпали с теоретическими с погрешностью 0,5 мм. Это доказало правильность результатов, полученных теоретическим путем.

\section{Заключение}

Разработана лабораторная установка, имеющая силовую характеристику с участком квазинулевой жесткости для оценки теоретических результатов. Основным исследуемым элементом лабораторной установки является пневматическая пружина, внутри которой расположен пакет из пяти последовательно установленных тарельчатых пружин.

Экспериментально подобраны параметры тарельчатых пружин таким образом, чтобы получить силовую характеристику с участком квазинулевой жесткости при заданном давлении в подпоршневой полости цилиндра.

Исследована силовая характеристика пневмокомпенсатора с квазинулевой жесткостью электроцентробежного погружного насоса. Получена силовая характеристика пакета последовательно соединенных тарельчатых пружин, имеющая участок с отрицательной жесткостью.
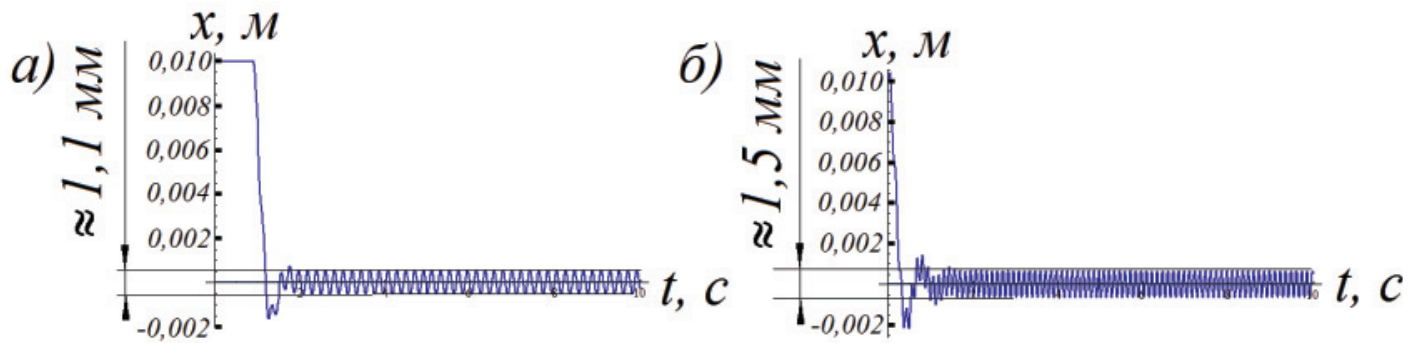

Рис. 10. Колебания поршня установки при значениях возмушаюшей силь в виде $F_{0}=p^{2} e\left(m_{1}+m_{2} / 2\right)$, полученные решением дифференииального уравнения (7): e=10 мл- эксиентриситет; $\left.\left(m_{1}+m_{2} / 2\right)=0,100 \kappa 2 ; a\right) p=30 c^{-1}$, размах колебаний $\approx 1,1$ мл; б) $p=50 c^{-1}$, разлах колебаний $\approx 1,5 \mathrm{mм}$

Fig. 10. Oscillations of the installation piston with the values of the disturbing force in the form of $F_{0}=p^{2} e\left(m_{1}+m_{2} / 2\right)$, obtained by solving the differential equation ( 7$): e=10 \mathrm{~mm}$ - eccentricity; $\left.\left(m_{1}+m_{2} / 2\right)=0,100 \mathrm{~kg} ; a\right) p=30 \mathrm{~s}^{-1}$, vibration swing $\left.\approx 1,1 \mathrm{~mm} ; \sigma\right) p=50 \mathrm{~s}^{-1}$, vibration swing $\approx 1,5 \mathrm{~mm}$ 


\section{СПИСОК ЛИТЕРАТУРЫ}

1. Исследование эффективности поршневого компенсатора УЭЦН с квазинулевой жесткостью. Ч. 2: Характеристика пневмокомпенсатора с квазинулевой жесткостью / Е.Б. Думлер, А.Н. Зотов, К.Р. Уразаков, Р.И. Вахитова // Нефтегазовое дело: науч. журн. УГНТУ. - 2017. - Т. 15. - № 3. C. $112-119$.

2. Думлер Е.Б., Зотов А.Н, Уразаков К.Р. Моделирование работы пневмокомпенсатора с квазинулевой жесткостью в установке электропогружного центробежного насоса // Записки Горного института. - 2018. - Т. 229. - С. 70-76.

3. Баграмов Р.А. Буровые машины и комплексы. - М.: Недра, 1988. $-501 \mathrm{c}$

4. Valeev A.R., Zotov A.N., Tashbulatov R.R. Experimental study of low frequency vibration isolator with quasi-zero stiffness // Proc. of the $23^{\text {rd }}$ International Congress on Sound and Vibration. - Athens, Greece, 2016. - P. 1-7.

5. Zotov A.N., Valeev A.R., Harisov Sh.A. Application of Disk Springs for Manufacturing Vibration Isolators with Quasi-Zero Stiffness // Chemical and Petroleum Engineering. - 2015. V. 51. - № 3. - P. 194-200.

6. Adaptive negative stiffness: a new structural modification approach for seismic protection / D. Pasala, A. Sarlis, S. Nagarajaiah, D. Taylor // Journal of structural Engineering. - 2013. V. 139. - № 7. - P. 1112-1123.

7. Carrella A., Friswell M.A. A passive vibration isolator incorporating a composite bistable plate // IPACS 0pen Access Electronic Library. $6^{\text {th }}$ Euromech Nonlinear Dynamics Conference, ENOC. St. Peterburg, 2008.

8. Carrella A. Passive vibration isolators with high-static-low-dynamic-stiffness: Ph.D. Thesis. - Soton, 2008. - 226 p.

9. Ibrahim R.A. Recent advances in nonlinear passive vibration isolators // Journal of Sound and Vibration. - 2008. - V. 314. № 3-5. - P. 371-452.
10. Le T.D., Ahn K.K. A vibration isolation system in low frequency excitation region using negative stiff-ness structure for vehicle seat // Journal of Sound and Vibration,. - 2011. - V. 330. № 26. - P. 6311-6335.

11. Liu X., Huang X., Hua H. On the characteristics of a quasi-zero stiffness isolator using Euler buck-led beam as negative stiffness corrector // Journal of Sound and Vibration. - 2013. - V. 332. № 14. - P. 3359-3376.

12. Mizuno T., Toumiya T., Takasaki M. Vibration Isolation System Using Negative Stiffness // JSME International Journal, Series C. - 2003. - V. 46. - № 3. - P. 807-812.

13. Wang Y.C., Lakes R.S. Extreme stiffness systems due to negative stiffness elements // American Journal of Physics. - 2004. V. 72. - № 1. - P. 40-50.

14. Valeev A.R., Kharisov Sh. Application of Vibration Isolators with a Low Stiffness for the Strongly Vibrating Equipment // Procedia Engineering. - 2016. - V. 150. - P. 641-646.

15. Алабужев П.М., Гритчин А.А., Ким Л.И. Виброзащитные системы с квазинулевой жесткостью / под ред. К.М. Рагульскиса. - Л.: Машиностроение, 1986. - 96 с.

16. Скважинная насосная установка: пат. Рос. Федерация № 2641812; заявл. 20.02.2017; опубл. 22.01.2018. Бюл. № 3. $14 \mathrm{c.}$

17. Flex пластик REC URL: https://rec3d.ru/shop/plastik-dlya-3dprinterov/flex/flex-plastik-rec-1-75mm-rozovyj/ (дата обращения: 15.09.2017).

18. Пановко Я.Г. Введение в теорию механического удара. - М.: Наука, 1977. - 232 с.

19. Ногид Л.М. Теория подобия и размерностей. - Л.: Судпромгиз, 1959. $-98 \mathrm{c}$

20. Андреева Л.Е. Упругие элементы приборов: 2 изд., перераб. и доп. - М.: Машиностроение, 1981. - 391 с.

Поступила 19.04.2018 г.

\section{Информация об авторах}

Зотов А.Н., доктор технических наук, профессор кафедры механики и конструирования машин Уфимского государственного нефтяного технического университета.

Думлер Е.Б., соискатель кафедры машин и оборудования нефтегазовых промыслов Уфимского государственного нефтяного технического университета.

Уразаков $\boldsymbol{K} . \boldsymbol{P . , ~ д о к т о р ~ т е х н и ч е с к и х ~ н а у к , ~ п р о ф е с с о р ~ к а ф е д р ы ~ м а ш и н ~ и ~ о б о р у д о в а н и я ~ н е ф т е г а з о в ы х ~ п р о м ы - ~}$ слов Уфимского государственного нефтяного технического университета.

Baxuтова P.И., кандидат технических наук, доцент кафедры электро- и теплоэнергетики Альметьевского государственного нефтяного института.

Думлер О.Ю., инженер-механик, директор по качеству и логистике промышленного предприятия по производству ЭЦН ООО «Новые технологии».

Тугунов П.М., аспирант кафедры машин и оборудования нефтегазовых промыслов Уфимского государственного нефтяного технического университета. 


\title{
EXPERIMENTAL STUDIES OF OPERATION OF A PNEUMATIC SPRING PRESSURE COMPENSATOR WITH QUASI-ZERO STIFFNESS
}

\author{
Alexey N. Zotov', \\ a-zot2@yandex.ru \\ Elena B. Dumler', \\ dumler08@mail.ru
}

Kamil R. Urazakov', UrazakK@mail.ru

\section{Roza I. Vakhitova ${ }^{2}$} roza-w@mail.ru

Oleg Yu. Dumler ${ }^{3}$, mail@ntehn.ru

\author{
Pavel M. Tugunov', \\ info@rusoil.net \\ 1 Ufa State Oil Technical University, \\ 1, Kosmonavtov street, Ufa, 450062, Russia. \\ ${ }^{2}$ Almetyevsk State Oil Institute, \\ 2, Lenin street, Almetyevsk, 423450, Russia. \\ ${ }^{3}$ LLC «New Technologies», \\ 168 c, Karl Marx street, Chistopol, 422980, Russia.
}

The relevance of the research is caused by vibration negative effect on the elements in the installation of the electric centrifugal pump. The presence of vibration increases the number of premature failures. One of the reasons for the vibrational effects on the electric centrifugal pump unit is the pressure drop at the pump discharge, and the frequencies of such fluctuations are small.

The main aim of the research is to develop a laboratory installation simulating oscillatory processes occurring on the discharge line of an electric centrifugal submersible pump in a well.

Objects: a model of a pneumatic spring pressure compensator with quasi-zero stiffness, structurally representing a pneumatic spring, inside of which there is a package of five sequentially installed disc springs.

Methods: numerical solutions of differential equations; theory of oscillations, mathematical analysis, mathematical statistics, methods of mathematical modeling.

Results. To confirm the theoretical results obtained by calculation the authors have developed the laboratory facility, which allows studying the system with quasi-zero stiffness. This is a set of pneumatic spring having a force characteristic with a working area of positive stiffness and a package of sequentially connected disc springs having a force characteristic with a working area of negative stiffness. The parameters of the disk springs were experimentally selected in such a way as to obtain a power characteristic with a region of quasi-zero stiffness at a given pressure in the cylinder sub-piston cavity. To ensure the necessary stability of the system to small changes with the help of the analysis of power characteristics, an optimum number of disk springs is obtained. The authors investigated the power characteristic of a pneumatic compensator with quasi-zero stiffness on the discharge line of an electric centrifugal submersible pump. The result of the research on the laboratory installation was the coincidence with the given error of the oscillation amplitude measurements and theoretically predicted results, which allowed proving the correctness of theoretical conclusions.

\section{Key words:}

Electric submersible pump, laboratory installation, pneumatic spring pressure compensator, pneumocompensator, pneumospring, quasi-zero stiffness, negative stiffness, dish-shaped springs.

\section{REFERENCES}

1. Investigation of the ESP piston compensator efficiency with quasi-zero rigidity. Part 2: Characteristics of the pneumatic compensator with quasi-zero rigidity / E.B. Dumler, A.N. Zotov, K.R. Urazakov, R.I. Vakhitova. Oil and gas business: scientific: journal UGNTU, 2017, vol. 15, no. 3, pp. 112-119. In Rus.

2. Dumler E.B., Zotov A.N., Urazakov K.R. Simulation of the work of a pneumatic compensator with quasi-zero rigidity in an electric submersible centrifugal pump installation. Notes of the Mining Institute, 2018, vol. 229, pp. 70-76. In Rus.
3. Bagramov R.A. Burovye mashiny i kompleksy [Drilling machines and complexes]. Moscow, Nedra Publ., 1988. 501 p.

4. Valeev A.R., Zotov A.N., Tashbulatov R.R. Experimental study of low frequency vibration isolator with quasi-zero stiffness. Proc. of the $23^{\text {rd }}$ International Congress on Sound and Vibration. Athens, Greece, 2016. pp. 1-7.

5. Zotov A.N., Valeev A.R., Harisov Sh.A. Application of Disk Springs for Manufacturing Vibration Isolators with Quasi-Zero Stiffness. Chemical and Petroleum Engineering, 2015, vol. 51, no. 3, pp. 194-200. 
6. Pasala D., Sarlis A., Nagarajaiah S., Taylor D. Adaptive negative stiffness: a new structural modification approach for seismic protection. Journal of structural Engineering, 2013, vol. 139, no. 7, pp. 1112-1123.

7. Carrella A., Friswell M.A. A passive vibration isolator incorporating a composite bistable plate. IPACS Open Access Electronic Library. $6^{\text {th }}$ Euromech Nonlinear Dynamics Conference, ENOC. St. Petersburg, 2008.

8. Carrella A. Passive vibration isolators with high-static-low-dynamic-stiffness. Ph.D. Thesis. Soton, 2008. 226 p.

9. Ibrahim R.A. Recent advances in nonlinear passive vibration isolators. Journal of Sound and Vibration, 2008, vol. 314, no. 3-5, pp. $371-452$.

10. Le T.D., Ahn K.K. A vibration isolation system in low frequency excitation region using negative stiff-ness structure for vehicle seat. Journal of Sound and Vibration, 2011, vol. 330, no. 26, pp. 631-635.

11. Liu X., Huang X., Hua H. On the characteristics of a quasi-zero stiffness isolator using Euler buck-led beam as negative stiffness corrector. Journal of Sound and Vibration, 2013, vol. 332, no. 14, pp. 3359-3376.

12. Mizuno T., Toumiya T., Takasaki M. Vibration Isolation System Using Negative Stiffness. JSME International Journal, Series C, 2003, vol. 46, no. 3, pp. 807-812.

13. Wang Y.C., Lakes R.S. Extreme stiffness systems due to negative stiffness elements. American Journal of Physics, 2004, vol. 72 , no. 1 , pp. 40-50.

\section{Information about the authors}

Alexey N. Zotov, Dr. Sc., professor Ufa State Oil Technical University.

Elena B. Dumler, graduate student, Ufa State Oil Technical University.

Kamil R. Urazakov, Dr. Sc., professor, Ufa State Oil Technical University.

Roza I. Vakhitova, Cand. Sc., associate professor, Almetyevsk State Oil Institute.

Oleg Yu. Dumler, engineer-mechanic, director of quality and logistics, LLC «New Technologies» LLC «New Technologies».

Pavel M. Tugunov, graduate student, Ufa State Oil Technical University.
14. Valeev A.R., Kharisov Sh. Application of Vibration Isolators with a Low Stiffness for the Strongly Vibrating Equipment. Procedia Engineering, 2016, vol. 150, pp. 641-646.

Alabuzhev P.M., Gritchin I.I. Kim L.I. Vibrozashchitnye sistemy $s$ kvazinulevoy zhestkostyu [Vibroprotective system with quasizero stiffness]. Ed. by K.M. Ragulskis. Leningrad, Mashinostroe-

16. Urazakov K.P., Dumler E.B., Zotov A.N., Dumler O.Yu. Skvazhinnaya nasosnaya ustanovka [Downhole pump installation]. Patent RF, no. 2641812, 2018.

17. Flex plastic REC. Available at: https: //rec3d.ru/shop/plastikdlya-3d-printerov/flex/flex-plastik-rec-1-75mm-rozovyj/ (accessed 15 September 2017).

18. Panovko Ya.G. Vvedenie v teoriyu mekhanicheskogo udara [Introduction to the theory of mechanical blow]. Moscow, Nauka Publ., 1977. $232 \mathrm{p}$.

. Nogid L.M. Teoriya podobiya i razmernostey [The theory of similarity and dimensions]. Leningrad, Sudpromgiz Publ., 1959. $98 \mathrm{p}$.

Andreeva L.E. Uprugie elementy i pribory [Elastic elements and devices]. Moscow, Mashinostroenie Publ., 1981. 391 p.

Received: 19 April 2018. nie Publ., $1986.96 \mathrm{p}$ 